Research Paper

\title{
Long-term Patterns of Regional Failure for Nasopharyngeal Carcinoma following Intensity-Modulated Radiation Therapy
}

\author{
Fen Xue1,2, Chaosu $\mathrm{Hu}^{1,2}$, Xiayun $\mathrm{He}^{1,2 \llbracket}$ \\ 1. Department of Radiation Oncology, Fudan University Shanghai Cancer Center, Shanghai, China. \\ 2. Department of Oncology, Shanghai Medical College, Shanghai, China. \\ $\triangle$ Corresponding author: Xiayun He. Mailing address: Department of Radiation Oncology, Room 704, Building 1, Dong'an Road 270, Fudan University \\ Shanghai Cancer Center, Shanghai, China. Telephone: +86-13917564793; E-mail: 15211230040@fudan.edu.cn. \\ (c) Ivyspring International Publisher. This is an open access article distributed under the terms of the Creative Commons Attribution (CC BY-NC) license \\ (https://creativecommons.org/licenses/by-nc/4.0/). See http://ivyspring.com/terms for full terms and conditions.
}

Received: 2016.10.09; Accepted: 2016.12.23; Published: 2017.03.15

\begin{abstract}
Purpose: To analyze the long-term patterns of regional failure following intensity-modulated radiotherapy (IMRT) for nasopharyngeal carcinoma (NPC).

Methods: From January 2005 to December 2010, 275 non-metastatic NPC patients treated with IMRT were retrospectively enrolled. Patients staged as II (lymph nodes measuring 4 or more $\mathrm{cm}$ in diameter), III or IV also received chemotherapy. Failures were assessed as in-field or out-field relative to the pretreatment planning computed tomography data sets. Univariate and multivariate analyses were performed with Cox proportional hazards model to analyze the effect of various prognostic factors on regional failure-free survival (RFFS) and overall survival (OS).

Results: During a median follow-up of 71 months, the RFFS and OS rates were $94.3 \%$ and $83.9 \%$, respectively. Seventeen patients developed regional failures, of which 16 were in-field; one patient showed an out-field failure in the parotid gland, and no recurrences were seen for level lb. Failures in level II and in the retropharyngeal area accounted for $70.6 \%(12 / 17)$ and $52.9 \%(9 / 17)$ of all failures, respectively. The 5-year RFFS rates for patients with classifications of N0-1 and N2-3 were $98.5 \%$ and $90.2 \%$, respectively $(p=0.001)$. Multivariate analysis showed that $\mathrm{N}$ stage was the only independent prognostic predictor of RFFS (HR 7.363, 95\% Cl 1.516-35.756, $p=0.013$ ).

Conclusions: The regional failure of NPC after treatment with IMRT is uncommon but is significantly higher in N2-3 patients than in N0-1 patients. In-field failures represent the main pattern of regional recurrence and are most often detected in level II and in the retropharyngeal area, while out-field failure is rare. Close attention should be directed to NPC patients with advanced $\mathrm{N}$ stages.
\end{abstract}

Key words: nasopharyngeal carcinoma; intensity-modulated radiotherapy; patterns of regional failure; $\mathrm{N}$ stage

\section{Introduction}

Nasopharyngeal carcinoma (NPC) has a high propensity for regional node metastasis [1]. Owing to its anatomical location and histological characteristics, radiotherapy (RT) with or without chemotherapy has been the mainstay of definitive treatment for NPC [2]. A meta-analysis [3] based on 13 clinical trials revealed that lymphatic spread in the cervical nodal chain from NPC primarily follows an orderly fashion with infrequent skip metastases. The authors of the meta-analysis also reported that the most commonly affected lymph node regions include the retropharyngeal nodes and the level II nodes, whereas the least commonly affected lymph node regions include level I, level VI, and parotid nodes. These findings are important for the selection of an optimal treatment strategy and for the delineation of 
subclinical regional disease.

During the conventional two-dimensional RT era, the fields of RT for NPC typically encompassed the primary disease and affected neck nodes, as well as the entire draining lymphatic region of the neck; co-irradiation of the parotid glands was thus unavoidable. However, such a strategy might represent over-treatment and may lead to substantial and serious toxicities owing to the large field involvement of the neck, irrespective of nodal status $[4,5]$. With the advent of intensity-modulated radiotherapy (IMRT), treatment delivery can be selective and accurate, targeted to the nodal spread patterns of NPC. For instance, treatment can include high-risk lymph node regions while sparing low-risk lymph node regions. Although a series of studies reported satisfactory regional control and decreased late normal tissue toxicities for NPC patients after IMRT with selective neck irradiation, geographic misses still occur and may cause unexpected regional failures, such as recurrence in parotid nodes [6-10]. Therefore, in this study, we retrospectively analyzed the 5-year treatment results of patients with NPC who were treated with IMRT to identify long-term patterns of regional failure.

\section{Materials and Methods}

\section{Patients and pretreatment evaluations}

From January 2005 to December 2010, 275 newly diagnosed, non-metastatic, and histologically confirmed NPC patients who were treated with definitive IMRT at the Shanghai Cancer Center of Fudan University were enrolled in this study. All patients underwent disease restaging using the 2010 staging system of American Joint Committee on Cancer (AJCC) [11]. The initial evaluation included medical history and physical examination, blood chemistry tests, chest X-ray/computed tomography (CT), abdomen ultrasound/CT, enhanced magnetic resonance imaging (MRI) of the nasopharynx and neck, nasopharyngoscopy, and bone emission CT. Additional investigations were performed only in patients with suspicious findings. Dental extraction, if deemed necessary, was performed before radiation therapy. This study was approved by our institution's ethics committee (Fudan University Shanghai Cancer Center Institutional Review Board, reference number 090371-5). All patients provided informed written consent to participate in this study.

\section{Radiotherapy}

All patients were treated in the supine position using a thermoplastic mask and received IMRT with 6 MV X-ray. Intravenous contrast-enhanced CT using a slice thickness of $5 \mathrm{~mm}$ was performed for RT planning. The CT data were imported into the treatment-planning system for treatment design.

The primary gross tumor volume (GTV) and the involved lymph nodes included all gross tumors found during the clinical and imaging examination. The clinical target volume (CTV) included the nasopharynx, the retropharyngeal lymph node, the skull base, the anterior third of the clivus, the pterygoid fossa, the parapharyngeal space, the inferior sphenoid sinus, the posterior third of the nasal cavity and the maxillary sinus (the whole sphenoid sinus and clivus should be covered for stage T3 and T4 disease), and the drainage region of the upper neck (levels II, III and Va in N0 patients and levels $\mathrm{II}-\mathrm{Vb}$ in N1-N3 patients). Neck level Ib was excluded from the CTV in patients without node involvement in level Ib. Critical normal structures, including the brainstem, spinal cord, optic nerves, chiasm, lens, eyeballs, temporal lobes, parotid glands, and larynx, were carefully delineated.

The prescription doses were 66-70.4 Gy/30-32 fractions to the GTV in the nasopharynx, 66 Gy to the positive neck nodes, 60 Gy to the high-risk CTV and 54 Gy to the low-risk CTV. All patients were irradiated with 1 fraction each day for 5 days per week.

\section{Chemotherapy}

All of the patients at stage II (lymph nodes measuring 4 or more $\mathrm{cm}$ in diameter), III or IV received platinum-based chemotherapy. A total of 241 $(87.6 \%)$ patients received chemotherapy; concurrent and nonconcurrent (neoadjuvant \pm adjuvant) chemotherapy was administered to 31 and 210 patients, respectively.

\section{Assessment and follow-up}

Patients were evaluated weekly during radiation therapy. After treatment completion, follow-up assessments were conducted every 3 months for the first 2 years, every 6 months from the third through the fifth year, and annually thereafter. Each follow-up included medical history, physical examination, and nasopharyngoscopy. An enhanced MRI of the nasopharynx and neck areas was performed every 6 to 12 months after treatment. Chest $\mathrm{X}$-ray and abdominal ultrasonography were conducted once yearly. Additional tests were ordered whenever there was any clinical indication.

Adverse events related to chemotherapy and radiotherapy were graded by the National Cancer Institute Common Toxicity Criteria, version 3.0 [12] and by the Radiation Therapy Oncology Group criteria [13], respectively. The assessment of tumor response was based on MRI findings according to the 
Response Evaluation Criteria for Solid Tumors criteria [14].

\section{Definition of failure site}

Regional failure was attributed to different nodal levels, according to the updated consensus on the delineation of neck node levels proposed by Gregoire et al. [15]. The exact site and extent of each tumor were compared with the pretreatment planning CT data sets, with a focus on the $95 \%$ isodose lines. Recurrent nodes were defined as in-field if $95 \%$ of the recurrent node volume was within the $95 \%$ isodose lines, as marginal field if $20 \%$ to $95 \%$ of the recurrent node volume was within the $95 \%$ isodose lines, or as out-field if less than $20 \%$ of the recurrent node volume was inside the $95 \%$ isodose lines [16].

\section{Statistics}

All statistical analyses were performed with SPSS software (version 22.0). Local failure-free survival (LFFS), regional failure-free survival (RFFS), distant failure-free survival (DFFS) and overall survival (OS) were estimated using the Kaplan-Meier method. LFFS, DFFS, and RFFS were measured from the date of treatment to the documentation of treatment failure or to the last follow-up, and OS was measured from the date of treatment to the date of death or the last follow-up. Differences in survival were assessed with a log-rank test. Multivariate analysis was performed using the Cox proportional hazards model. A p value of less than 0.05 was considered to indicate statistical significance. The survival curves were plotted using GraphPad Prism 6.0 software.

\section{Results}

\section{Patient characteristics}

A total of 275 NPC patients with a definite pathological diagnosis (WHO II-III) were analyzed. The patients included 209 males and 66 females with a median age of 49 (16-73) years. All patients completed IMRT, except for two patients who discontinued radiotherapy owing to Grade III mucositis or for personal reasons at doses of $59.4 \mathrm{~Gy} / 27 \mathrm{~F}$ and 63.8 Gy/29F. The characteristics of the patients are listed in Table 1. The distributions of the $\mathrm{T}$ and $\mathrm{N}$ classifications according to the AJCC staging system are listed in Table 2.

\section{Patterns of regional failure}

Among the enrolled patients, 17 exhibited regional recurrence. The median interval from the start of treatment to the diagnosis of nodal failure was 29 (8-79) months. Table 3 shows the patterns of regional failure with respect to radiation field coverage at each level. Considering multilevel regional recurrences, the most frequent recurrence sites were level II and the retropharyngeal area, accounting for $70.6 \%(12 / 17)$ and $52.9 \%(9 / 17)$ of all cases, respectively. Other recurrent nodal levels included level III in 5 patients and level IV in 4 patients. All but 1 regional failure developed in-field; the exception occurred in a patient who displayed recurrent disease in the parotid gland region (out-field). No level Ib failures were detected. Of the five patients with multilevel recurrent nodes in the neck, two had a conglomerate of at least three nodes in the ipsilateral neck before initial treatment, one had bulky nodes at level II, and the other two had bilateral multilevel nodes and supraclavicular lymph nodes before the initial treatment. The patient who failed in the spared region of the parotid gland 28 months after IMRT had bilateral multilevel lymph nodes and right retropharyngeal lymph nodes before the initial treatment, and no evidence of nodules was observed in pretreatment MRI images. Eventually, 10 patients died of regional failure; 5 of them exhibited distant metastasis.

Table 1. Patient characteristics

\begin{tabular}{lc}
\hline Characteristics & No. of patients (\%) \\
\hline Age (years) & $143(52)$ \\
$<50$ & $132(48)$ \\
$\geq 50$ & \\
Gender & $209(76)$ \\
Male & $66(24)$ \\
Female & \\
KPS & $16(5.8)$ \\
70 & $118(42.9)$ \\
80 & $141(51.3)$ \\
90 & \\
Regional lymph node & $55(20)$ \\
FNAC & $17(6.2)$ \\
Biopsy & $203(73.8)$ \\
None & $178(64.7)$ \\
Metastasis to retropharyngeal lymph nodes & \\
Lymph node liquefaction & $109(39.6)$ \\
Yes & $166(60.4)$ \\
No & \\
AJCC Stage & $16(5.8)$ \\
I & $54(19.6)$ \\
II & $102(37.1)$ \\
III & $103(37.5)$ \\
IV & \\
Chemotherapy & $241(87.6)$ \\
Yes & $34(12.4)$ \\
No &
\end{tabular}

Of the 17 patients with regional recurrence, none had N0 disease, while 2, 9 and 6 patients had N1, N2 and N3 disease, respectively. The overall 5-year LFFS rates for patients with classifications of N0-1 and N2-3 were $92.3 \%$ and $93.8 \%$, respectively $(p=0.713$; Figure 
1A). The overall 5 -year RFFS rates for patients with classifications of N0-1 and N2-3 were $98.5 \%$ and $90.2 \%$, respectively $(\mathrm{p}=0.001$; Figure $1 \mathrm{~B})$. The overall 5 -year DFFS rates for patients with classifications of N0-1 and N2-3 were $93.2 \%$ and $78.6 \%$, respectively (p $<0.001$; Figure 1C). The overall 5-year OS rates for patients with classifications of N0-1 and N2-3 were $91.7 \%$ and $76.4 \%$, respectively ( $p=0.001$; Figure $1 D)$.

\section{Second primary cancers}

A total of 13 patients developed second primary cancer after IMRT. One patient was diagnosed with acinic cell carcinoma of the parotid 96 months after IMRT, and seven patients developed lung cancer 9-78 months after radiotherapy, as confirmed by pathology. The remaining patients developed neck pleomorphic sarcoma, esophageal cancer, thyroid adenocarcinoma, sigmoid carcinoma or melanoma 27-97 months after IMRT.

Table 2. Distribution of patients according to AJCC stage

\begin{tabular}{|c|c|c|c|c|c|}
\hline \multirow[t]{2}{*}{ Stage } & \multicolumn{5}{|c|}{ No. of patients (\%) } \\
\hline & $\mathrm{T} 1$ & $\mathrm{~T} 2$ & T3 & $\mathrm{T} 4$ & Total \\
\hline No & $16(5.8)$ & $7(2.5)$ & $9(3.3)$ & $10(3.6)$ & $42(15.3)$ \\
\hline N1 & $17(6.2)$ & $30(10.9)$ & $22(8.0)$ & $22(8.0)$ & $91(33.1)$ \\
\hline N2 & $9(3.3)$ & $30(10.9)$ & 32 (11.6) & $24(8.7)$ & $95(34.5)$ \\
\hline N3 & $9(3.3)$ & $23(8.4)$ & $9(3.3)$ & $6(2.2)$ & 47 (17.1) \\
\hline Total & $51(18.6)$ & $90(32.7)$ & $72(26.2)$ & $62(22.5)$ & $275(100.0)$ \\
\hline
\end{tabular}

A

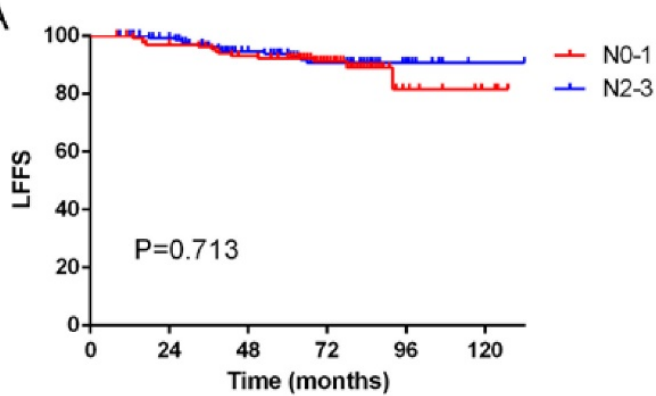

C

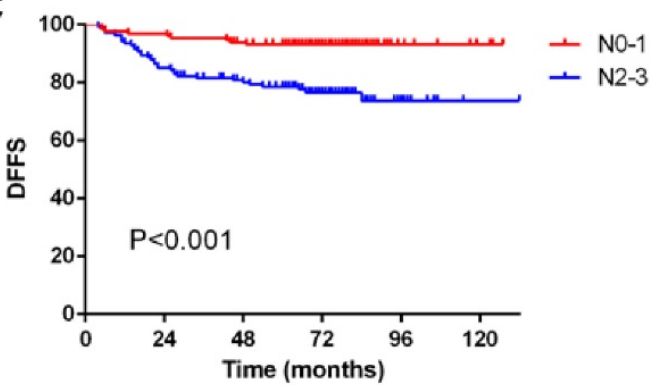

Table 3. Patterns of regional failure of the 17 patients

\begin{tabular}{|c|c|c|c|c|c|}
\hline $\begin{array}{l}\text { Patient } \\
\text { No. }\end{array}$ & AJCC stage & $\begin{array}{l}\text { Irradiation } \\
\text { level }\end{array}$ & Failure level & $\begin{array}{l}\text { Failure } \\
\text { pattern }\end{array}$ & $\begin{array}{l}\text { Survival } \\
\text { state }\end{array}$ \\
\hline 1 & T4N2, IV & II-V & Left II & In-field & Dead \\
\hline 2 & T4N3, IV & II-V & Left II & In-field & Dead* $^{*}$ \\
\hline 3 & T3N2, III & II-V & $\begin{array}{l}\text { Right PLN, Right } \\
\text { RLN, Right II-IV }\end{array}$ & Out-field & Dead* $^{*}$ \\
\hline 4 & T2N2, III & II-V & Left II-III, Right RLN & In-field & Dead \\
\hline 5 & T3N1, III & II-V & Left II, Right RLN & In-field & Alive \\
\hline 6 & T2N3, IV & II-V & Left II, Left RLN & In-field & Alive \\
\hline 7 & T2N3, IV & II-V & Right III, IV & In-field & Dead \\
\hline 8 & T3N2, III & II-V & Right II & In-field & Dead $^{*}$ \\
\hline 9 & T2N2, III & II-V & Right II & In-field & Dead $^{*}$ \\
\hline 10 & T3N1, III & II-V & Left II & In-field & Alive \\
\hline 11 & T2N3, IV & II-V & $\begin{array}{l}\text { Bilateral II-IV, Right } \\
\text { RLN }\end{array}$ & In-field & Dead \\
\hline 12 & T1N3, IV & II-V & Left II & In-field & Dead $^{*}$ \\
\hline 13 & T4N3, IV & II-V & Bilateral II-IV & In-field & Alive \\
\hline 14 & T2N2, III & II-V & Right RLN & In-field & Alive \\
\hline 15 & T2N2, III & II-V & Right RLN & In-field & Dead \\
\hline 16 & T2N2, III & II-V & Left RLN & In-field & Alive \\
\hline 17 & T4N2, IV & II-V & Right RLN & In-field & Alive \\
\hline
\end{tabular}

*Died of regional recurrence accompanied by distant metastasis.

PLN, parotid gland lymph node; RLN, retropharyngeal lymph node.
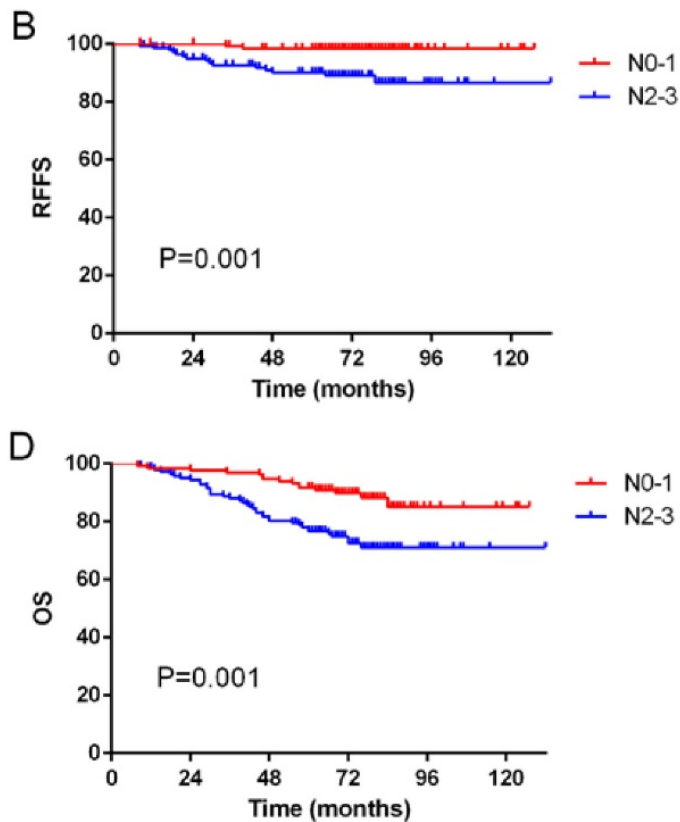

Figure 1. Kaplan-Meier curves showing (A) local failure-free survival (LFFS), (B) regional failure-free survival (RFFS), (C) distant failure-free survival (DFFS) and (D) overall survival (OS) in N0-1 and N2-3 patients. 
Table 4. Details of patients who developed treatment failure

\begin{tabular}{lc}
\hline Site & No. of patients (\%) \\
\hline Local only & $15(5.5)$ \\
Regional only & $6(2.2)$ \\
Distant only & $33(12.0)$ \\
Local and regional & $5(1.8)$ \\
Local and distant & $3(1.1)$ \\
Regional and distant & $5(1.8)$ \\
Local, regional and distant & $1(0.4)$ \\
Overall distant failure & $42(15.3)$ \\
Distant failure sites & \\
Bone only & $7(2.5)$ \\
Liver only & $5(1.8)$ \\
Lung only & $5(1.8)$ \\
Lung and mediastinum & $13(4.7)$ \\
Other location & $4(1.5)$ \\
Multiple locations & $8(2.9)$ \\
\hline
\end{tabular}

\section{Treatment outcomes}

With a median follow-up of 71 (8-132) months, 68 patients $(24.7 \%)$ had developed treatment failure at their final follow-up visit. Among these patients, 15, 6, and 33 had developed local failure, regional failure, and distant metastasis, respectively; 5 had developed locoregional failure, 3 had developed distant metastasis and failure at the primary site, 5 had developed distant metastasis and failure at the nodal site, and 1 had developed distant metastasis and failure at the primary and nodal sites. Additional details are listed in Table 4 . By the final follow-up, 53 patients $(19.3 \%)$ had died of treatment failure. Among these patients, 11, 24, 4, and 2 patients died of locoregional failure, distant metastasis, second primary cancers, and complications, respectively; 7 patients died of locoregional failure and distant metastasis, 2 patients died of non-neoplastic diseases, and 3 patients died of unknown causes. The overall 5-year LFFS, RFFS, DFFS, and OS rates were 93.0\%, $94.3 \%, 85.7 \%$, and $83.9 \%$, respectively (Figure 2 ).

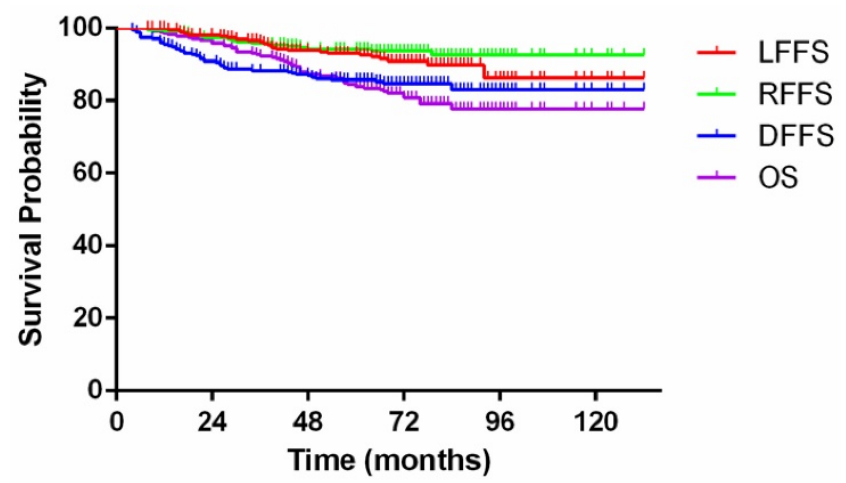

Figure 2. Kaplan-Meier curves showing local failure-free survival (LFFS), regional failure-free survival (RFFS), distant failure-free survival (DFFS) and overall survival (OS) for patients with nasopharyngeal carcinoma.

\section{Prognostic factors}

Univariate analysis of the entire cohort was performed using the log-rank test to examine the impacts of various potential prognostic factors, including age, gender, metastasis to retropharyngeal lymph nodes, $\mathrm{N}$ stage, lymph node liquefaction, and the acceptance of lymph node biopsy or fine-needle aspiration, on RFFS, DMFS, and OS (Table 5). The analysis showed that $\mathrm{N}$ stage was significantly associated with RFFS. Metastasis to retropharyngeal lymph nodes, $\mathrm{N}$ stage, lymph node liquefaction, and the acceptance of lymph node biopsy or fine-needle aspiration were significantly associated with DFFS. Age, $\mathrm{N}$ stage and lymph node liquefaction were significantly associated with OS.

Multivariate analysis with the Cox proportional-hazards model showed that $\mathrm{N}$ stage was the only independent prognostic predictor of RFFS (HR 7.363, 95\% CI 1.516-35.756, p = 0.013). Lymph node liquefaction and $\mathrm{N}$ stage were independent prognostic predictors of DMFS (HR 2.561, 95\% CI 1.256-5.042, $\mathrm{p}=0.009$; HR 2.250, 95\% CI 1.000-5.063, $\mathrm{p}$ $=0.050)$. Age, lymph node liquefaction, and $\mathrm{N}$ stage were independent prognostic predictors of OS (HR 2.177, 95\% CI 1.231-3.851, $\mathrm{p}=0.008$; HR 1.926, 95\% CI 1.056-3.513 $\mathrm{p}=0.032$; HR 2.111, 95\% CI 1.072-4.158, $\mathrm{p}$ $=0.031)$.

Table 5. Impact of prognostic factors on 5-year treatment results according to a univariate analysis

\begin{tabular}{|c|c|c|c|c|c|c|}
\hline Characteristic & RFFS (\%) & $P$ value & DFFS (\%) & $\begin{array}{l}P \\
\text { value }\end{array}$ & OS (\%) & $\begin{array}{l}\mathrm{P} \\
\text { value }\end{array}$ \\
\hline Age (years) & & 0.597 & & 0.991 & & 0.010 \\
\hline$<50$ & 95.6 & & 86.0 & & 89.4 & \\
\hline$\geq 50$ & 92.8 & & 85.4 & & 78.0 & \\
\hline Gender & & 0.520 & & 0.051 & & 0.051 \\
\hline Male & 93.4 & & 83.1 & & 81.1 & \\
\hline Female & 96.9 & & 93.9 & & 92.4 & \\
\hline Metastasis to RLN & & 0.093 & & 0.038 & & 0.055 \\
\hline No & 96.8 & & 91.7 & & 89.6 & \\
\hline Yes & 92.9 & & 82.4 & & 80.8 & \\
\hline Biopsy or FNA & & 0.709 & & 0.023 & & 0.632 \\
\hline No & 94.4 & & 88.5 & & 84.7 & \\
\hline Yes & 93.9 & & 77.8 & & 81.7 & \\
\hline N stage & & 0.001 & & $<0.001$ & & 0.001 \\
\hline N0-1 & 98.5 & & 93.2 & & 91.7 & \\
\hline $\mathrm{N} 2-3$ & 90.2 & & 78.6 & & 76.4 & \\
\hline $\begin{array}{l}\text { Lymph node } \\
\text { liquefaction }\end{array}$ & & 0.147 & & $<0.001$ & & $<0.001$ \\
\hline No & 95.7 & & 92.1 & & 90.3 & \\
\hline Yes & 91.8 & & 75.7 & & 73.8 & \\
\hline
\end{tabular}




\section{Discussion}

Regional node metastases are common in patients with NPC. Approximately $69.5 \%-86.4 \%$ of patients were reported to have regional lymph node metastases at the time of diagnosis [3, 9, 17]. Therefore, regional control is a major concern in NPC treatment. Li et al. [18] reported that $75 \%$ of their NPC patients had involved lymph nodes, and 7.7\% eventually underwent recurrence after treatment. A total of $84.7 \%(233 / 275)$ of the patients in our study presented with lymphadenopathy at the time of diagnosis, and only $6.2 \%(17 / 275)$ of the patients developed regional failure at a median follow-up time of 71 months. We achieved excellent 5-year regional control of $94.3 \%$, similar to $92.3 \%-93.0 \%$ reported by other IMRT studies $[8,19,20]$.

In the present study and in other NPC studies, a large proportion of regional recurrences occurred in-field. Kong et al. [21] analyzed 370 non-metastatic NPC patients treated with IMRT (with or without chemotherapy) and found regional relapses in 11 patients, of which $81.8 \%(9 / 11)$ were in-field. With a median follow-up of 70.4 months, $6.0 \%$ (10/165) of patients with NPC who had been treated with IMRT developed nodal relapse, and in-field failures accounted for $60 \%$ of all nodal failures, as reported by $\mathrm{Li}$ et al. [18]. The authors also demonstrated agreement between the phenomenon that most in-field failures occurred in level II and the distribution of pretreatment nodes in patients with NPC, as level II was the most frequently involved nodal group. Our results are in accordance with such findings, showing that in-field failures (94.1\%) represent the main pattern of regional recurrence. We also found that the most frequent failure sites were level II $(70.6 \%)$ and the retropharyngeal area (52.9\%); other recurrent nodal levels included level III in 5 patients and level IV in 4 patients. This finding suggested that the frequency of relapse in the jugular lymph node chains decreased in a cranio-caudal direction (level II 70.6\%, level III 29.4\%, and level IV $23.5 \%)$, similar to the distribution patterns of metastatic nodes at the time of diagnosis [17]. The predominance of in-field failures may result from nonuniform clonogenic cell density and radiosensitivity within the field, as most lymph nodes presented with liquefaction. Hypoxia often develops in liquefied areas because of a less-ordered, often chaotic, and leaky vascular supply compared with the vasculature in normal areas [22]. Hong et al. [23] presented a review of articles about tumor hypoxia and found that tumor hypoxia is common in NPC and is associated with disease progression and resistance to therapy, indicating that hypoxic radioresistance may be one cause of regional failure.
Though pre-existing metastasis at the periparotid area is rare, several cases have been reported of nodal recurrence in the parotids after IMRT for NPC. Li et al. [18] reported 4 cases with parotid nodal failure among 165 patients treated with parotid-sparing RT for NPC, and these cases accounted for all of the out-field failures and for $40 \%$ of the total nodal relapses. Three cases showed subcentimeter nodules at the same site in the parotid gland in which the recurrent nodes were located in the pretreatment MRI, despite a negative positron emission tomography (PET). Cao et al. [6] also reported 10 failures in the region of the parotid gland among 716 NPC patients after IMRT, of which $9(90 \%)$ had lateral retropharyngeal lymphadenopathy at the time of diagnosis. In the present study, only 1 patient failed in the spared region of the parotid gland with bilateral multilevel lymph nodes and right lateral retropharyngeal lymphadenopathy before the initial treatment, and no evident nodules were seen in pretreatment MRI images. These findings suggest to us that great attention must be paid to pretreatment images and to histopathological examination, especially when nonspecific periparotid nodules are seen; additionally, the presence of retropharyngeal lymphadenopathy or multilevel nodal disease at diagnosis may indicate a high risk of periparotid recurrence. Considering the proximity of the parotid gland to retropharyngeal lymphadenopathy and complex network of lymphatic channels and nodes, the recurrence may result from pretreatmentneglected micrometastasis and changes in lymphatic drainage after IMRT. Notably, 1 patient developed acinic cell carcinoma of the parotid gland as a second primary cancer in our study, suggesting a significant role for pathological diagnosis when abnormalities are seen in the parotid gland after IMRT.

The prophylactic irradiation of level $\mathrm{Ib}$ in patients with NPC remains controversial. Several studies reported that nodal metastases in level $\mathrm{Ib}$ were rare, with incidences ranging from 3 to $4.3 \%[3,17$, 24]. Li et al. [25] conducted a Phase 2 prospective study in 123 newly diagnosed Ib-negative NPC patients treated with IMRT. By the last follow-up visit, only two patients $(1.6 \%)$ had developed failures at level $\mathrm{Ib}$, so the authors suggested omitting elective neck irradiation of level $\mathrm{Ib}$ in Ib-negative patients. Results from other prospective and retrospective studies also showed that recurrence in unirradiated level $\mathrm{Ib}$ is acceptably low [26-28]. Our findings showed no nodal failures in level $\mathrm{Ib}$ in the absence of prophylactic level Ib irradiation, in accord with the above reports. Given the very low risks of nodal metastases and recurrence in level $\mathrm{Ib}$ among NPC cases, it is reasonable to postulate that prophylactic 
irradiation of level Ib may be unnecessary.

Prospective and retrospective studies [8, 9, 29]

have shown that $\mathrm{N}$ stage is an independent prognostic predictor of RFFS, DFFS and OS. Our results were in close agreement with these previous studies. In addition, the present study demonstrated that lymph node liquefaction was also an independent prognostic predictor of DFFS and OS, probably because the liquefied area is related to hypoxia and radioresistance, as illustrated by Hong et al. [23]. There were several limitations in this study. First, the chemotherapy regimens were not consistent. Second, the number of enrolled patients was relatively small. Therefore, prospective and randomized clinical trials with a larger cohort are needed for further research.

In conclusion, our study shows an excellent 5 -year regional control rate for NPC of $94.3 \%$ with IMRT. In-field failures (94.1\%) represented the main pattern of regional recurrence. Level II and the retropharyngeal area were the most frequent recurrence regions. Out-field failure was rare and usually occurred in the parotid gland region. No recurrences in level $\mathrm{Ib}$ were observed; thus, prophylactic irradiation of level $\mathrm{Ib}$ may be unnecessary. We also found that N2-3 stage patients had higher risks of developing regional recurrence. Further studies on the management of patients with advanced $\mathrm{N}$ stages are needed.

\section{Abbreviations}

IMRT: intensity-modulated radiotherapy; NPC: nasopharyngeal carcinoma; RT: radiotherapy; CT: computed tomography; MRI: magnetic resonance imaging; RFFS: failure-free survival; OS: overall survival; GTV: gross tumor volume; CTV: clinical target volume; LFFS: local failure-free survival; DFFS: distant failure-free survival; PET: positron emission tomography

\section{Acknowledgments}

We acknowledge the support of the Department of Radiation Oncology. The views expressed in this publication are those of the authors.

\section{Competing Interests}

The authors have declared that no competing interest exists.

\section{References}

[1] Sham JS, Choy D, Wei WI. Nasopharyngeal carcinoma: orderly neck node spread. Int J Radiat Oncol Biol Phys. 1990; 19: 929-933.

[2] Blanchard P, Lee A, Marguet S, et al. Chemotherapy and radiotherapy in nasopharyngeal carcinoma: an update of the MAC-NPC meta-analysis. Lancet Oncol. 2015; 16: 645-655.

[3] Ho FC, Tham IW, Earnest A, et al. Patterns of regional lymph node metastasis of nasopharyngeal carcinoma: a meta-analysis of clinical evidence. BMC Cancer. 2012; 12: 98 .
[4] Lee AW, Ng WT, Hung WM, et al. Major late toxicities after conformal radiotherapy for nasopharyngeal carcinoma-patient- and treatment-related risk factors. Int J Radiat Oncol Biol Phys. 2009; 73: 1121-1128.

[5] Lee AW, Poon YF, Foo W, et al. Retrospective analysis of 5037 patients with nasopharyngeal carcinoma treated during 1976-1985: overall survival and patterns of failure. Int J Radiat Oncol Biol Phys. 1992; 23: 261-270.

[6] Cao CN, Luo JW, Gao L, et al. Clinical characteristics and patterns of failure in the parotid region after intensity-modulated radiotherapy for nasopharyngeal carcinoma. Oral Oncol. 2013; 49: 611-614.

[7] Ou X, Zhou X, Shi Q, et al. Treatment outcomes and late toxicities of 869 patients with nasopharyngeal carcinoma treated with definitive intensity modulated radiation therapy: new insight into the value of total dose of cisplatin and radiation boost. Oncotarget. 2015; 6: 38381-38397.

[8] Peng G, Wang T, Yang KY, et al. A prospective, randomized study comparing outcomes and toxicities of intensity-modulated radiotherapy vs. conventional two-dimensional radiotherapy for the treatment of nasopharyngeal carcinoma. Radiother Oncol. 2012; 104: 286-293.

[9] Sun X, Su S, Chen C, et al. Long-term outcomes of intensity-modulated radiotherapy for 868 patients with nasopharyngeal carcinoma: an analysis of survival and treatment toxicities. Radiother Oncol. 2014; 110: 398-403.

[10] Li JG, Yuan X, Zhang LL, et al. A randomized clinical trial comparing prophylactic upper versus whole-neck irradiation in the treatment of patients with node-negative nasopharyngeal carcinoma. Cancer. 2013; 119: 3170-3176.

[11] Edge SB and Compton CC. The American Joint Committee on Cancer: the 7th edition of the AJCC cancer staging manual and the future of TNM. Ann Surg Oncol. 2010; 17: 1471-1474.

[12] Trotti A, Colevas AD, Setser A, et al. CTCAE v3.0: development of a comprehensive grading system for the adverse effects of cancer treatment. Semin Radiat Oncol. 2003; 13: 176-181.

[13] Cox JD, Stetz J and Pajak TF. Toxicity criteria of the radiation therapy oncology group (RTOG) and the European organization for research and treatment of cancer (EORTC). Int J Radiat Oncol Biol Phys. 1995; 31: 1341-1346.

[14] Eisenhauer E, Therasse P, Bogaerts J, et al. New response evaluation criteria in solid tumours: revised RECIST guideline (version 1.1). European journal of cancer. 2009; 45: 228-247.

[15] Gregoire V, Levendag P, Ang KK, et al. CT-based delineation of lymph node levels and related CTVs in the node-negative neck: DAHANCA, EORTC, GORTEC, NCIC,RTOG consensus guidelines. Radiother Oncol. 2003; 69: 227-236.

[16] Dawson LA, Anzai Y, Marsh L, et al. Patterns of local-regional recurrence following parotid-sparing conformal and segmental intensity-modulated radiotherapy for head and neck cancer. Int J Radiat Oncol Biol Phys. 2000; 46: 1117-1126.

[17] Wang $\mathrm{X}, \mathrm{Hu} \mathrm{C}$, Ying $\mathrm{H}$, et al. Patterns of lymph node metastasis from nasopharyngeal carcinoma based on the 2013 updated consensus guidelines for neck node levels. Radiother Oncol. 2015; 115: 41-45.

[18] Li JG, Venigalla P, Leeman JE, et al. Patterns of nodal failure after intensity modulated radiotherapy for nasopharyngeal carcinoma. Laryngoscope. 2016;

[19] Bayman E, Prestwich RJ, Speight R, et al. Patterns of failure after intensity-modulated radiotherapy in head and neck squamous cell carcinoma using compartmental clinical target volume delineation. Clin Oncol (R Coll Radiol). 2014; 26: 636-642.

[20] Wolden SL, Chen WC, Pfister DG, et al. Intensity-modulated radiation therapy (IMRT) for nasopharynx cancer: update of the Memorial Sloan-Kettering experience. Int J Radiat Oncol Biol Phys. 2006; 64: 57-62.

[21] Kong F, Ying H, Du C, et al. Patterns of local-regional failure after primary intensity modulated radiotherapy for nasopharyngeal carcinoma. Radiat Oncol. 2014; 9: 60.

[22] Janssen HL, Haustermans KM, Balm AJ, et al. Hypoxia in head and neck cancer: how much, how important? Head Neck. 2005; 27: 622-638.

[23] Hong B, Lui VW, Hashiguchi $M$, et al. Targeting tumor hypoxia in nasopharyngeal carcinoma. Head Neck. 2013; 35: 133-145.

[24] King AD, Ahuja AT, Leung SF, et al. Neck node metastases from nasopharyngeal carcinoma: MR imaging of patterns of disease. Head Neck. 2000; 22: 275-281.

[25] Li M, Huang XG, Yang ZN, et al. Effects of omitting elective neck irradiation to nodal Level IB in nasopharyngeal carcinoma patients with negative Level IB lymph nodes treated by intensity-modulated radiotherapy: a Phase 2 study. Br J Radiol. 2016; 89: 20150621

[26] Chen J, Ou D, He X, et al. Sparing level Ib lymph nodes by intensity-modulated radiotherapy in the treatment of nasopharyngeal carcinoma. Int J Clin Oncol. 2014; 19: 998-1004.

[27] Chen JZ, Le QT, Han F, et al. Results of a phase 2 study examining the effects of omitting elective neck irradiation to nodal levels IV and $\mathrm{Vb}$ in patients with $\mathrm{N}(0-1)$ nasopharyngeal carcinoma. Int J Radiat Oncol Biol Phys. 2013; 85: 929-934

[28] Zhang F, Cheng YK, Li WF, et al. Investigation of the feasibility of elective irradiation to neck level $\mathrm{Ib}$ using intensity-modulated radiotherapy for patients with nasopharyngeal carcinoma: a retrospective analysis. BMC Cancer. 2015; 15: 709 .

[29] Lee AW, Ng WT, Chan LL, et al. Evolution of treatment for nasopharyngeal cancer--success and setback in the intensity-modulated radiotherapy era. Radiother Oncol. 2014; 110: 377-384. 